\title{
The Uncertain Generalized Probabilistic Weighted Average and its Application in the Theory of Expertons
}

\author{
José M. Merigó, Montserrat Casanovas \\ Department of Business Administration, University of Barcelona \\ Av. Diagonal 690, 08034 Barcelona, Spain
}

\begin{abstract}
We introduce the uncertain generalized probabilistic weighted averaging (UGPWA) operator. It is an aggregation operator that unifies the probability and the weighted average in the same formulation considering the degree of importance that each concept has in the aggregation. Moreover, it is able to assess uncertain environments that can not be assessed with exact numbers but it is possible to use interval numbers. Thus, we can analyze imprecise information considering the minimum and the maximum result that may occur. We analyze the applicability of this new approach in a multiperson decision making problem by using the theory of expertons.
\end{abstract}

Keywords: Theory of expertons; Probabilistic weighted average; Interval numbers; Decision making

\section{Introduction}

Aggregation operators are very common in the literature [1,21-23,27]. They are very useful for assessing the available information in a more efficient way. One of the most common aggregation operators is the weighted average. It aggregates the information by giving different degrees of importance to the arguments considered in the problem. It has been used in an astonishingly wide range of applications [1,19,21]. Another very common aggregation operator is the probabilistic aggregation. It aggregates the information by using probabilities in the analysis. It has also been applied in a lot of applications $[8-9,26]$.

Another type of aggregation operators that are also becoming very popular in the literature are the generalized aggregation operators [1,10-13,15,24,28]. Its main characteristic is that it generalizes a wide range of aggregation operators by using generalized and quasiarithmetic means. Thus, we can include in the same formulation arithmetic aggregations, geometric aggregations or quadratic aggregations.

Recently, Merigó [7] has suggested a new approach that unifies the probability and the weighted average in the same formulation and considering the degree of importance of each concept in the aggregation. He has called it the probabilistic weighted averaging (PWA) operator. Its main advantage is that it can consider subjective and objective information in the same formulation. Thus, it is able to assess the information in a more complete way.
Usually, when dealing with these aggregation operators we assume that the information is clearly known and can be assessed with exact numbers. However, in real world problems, it is not so easy to assess the information because usually it is very complex and affected by different types of uncertainties. Thus, the use of exact numbers is not enough because it provides incomplete information and sometimes this may lead to wrong decisions. Therefore, in order to properly assess the information we need to use other techniques for representing the uncertainty in a more complete way such as the use of interval numbers. Note that by using interval numbers, the previous aggregation operators are known as the uncertain weighted average (UWA) and the uncertain probabilistic aggregation (UPA).

The aim of this paper is to present the uncertain generalized probabilistic weighted averaging (UGPWA) operator. It is a new aggregation operator that unifies the UWA and the UPA operator in the same formulation taking into account the degree of importance that each concept has in the analysis. Thus, it can represent the information considering subjective and objective perspectives and uncertain environments assessed with interval numbers. Moreover, it also uses generalized means that permits to provide a general framework that includes a wide range of particular cases including the UWA, the UPA, the uncertain generalized weighted average (UGWA), the uncertain generalized probabilistic aggregation (UGPA), the uncertain average (UA), the PWA operator, the uncertain PWA (UPWA) and many others.

We further generalize this approach by using quasiarithmetic means, obtaining the uncertain quasiarithmetic PWA (Quasi-UPWA) operator. It provides a more robust generalization that includes the UGPWA operator as a particular case.

We study the applicability of the new approach in a multi-person decision making problem regarding the selection of monetary policies by using the theory of expertons [5-6]. The theory of expertons extends the concept of probabilistic set [4] for uncertain environments that can be assessed with interval numbers. The main advantage of this approach is that we can analyze the information of the group in a more complete way considering all the individual opinions and producing a final single result.

This paper is organized as follows. In Section 2 we briefly describe some basic concepts. Section 3 introduces the UGPWA operator and Section 4 analyzes several families. Section 5 develops an application in decision making with the theory of expertons and Section 6 summarizes the main results of the paper. 


\section{Preliminaries}

In this Section we briefly review some basic concepts regarding the interval numbers, the uncertain generalized weighted average and the PWA operator.

\subsection{The interval numbers}

The interval numbers [17] are a very useful and simple technique for representing the uncertainty that has been used in a wide range of applications.

The interval numbers can be expressed in different forms. For example, if we assume a 4-tuple $\left(a_{1}, a_{2}, a_{3}\right.$, $\left.a_{4}\right)$, that is, a quadruplet; we could consider that $a_{1}$ and $a_{4}$ represents the minimum and the maximum of the interval number, and $a_{2}$ and $a_{3}$, the interval with the highest probability or possibility, depending on the use we want to give to the interval numbers. Note that $a_{1} \leq a_{2} \leq$ $a_{3} \leq a_{4}$. If $a_{1}=a_{2}=a_{3}=a_{4}$, then, the interval number is an exact number; if $a_{2}=a_{3}$, it is a 3-tuple known as triplet; and if $a_{1}=a_{2}$ and $a_{3}=a_{4}$, it is a simple 2-tuple interval number.

In the following, we are going to review some basic interval number operations as follows. Let $A$ and $B$ be two triplets, where $A=\left(a_{1}, a_{2}, a_{3}\right)$ and $B=\left(b_{1}, b_{2}, b_{3}\right)$. Then:

1) $A+B=\left(a_{1}+b_{1}, a_{2}+b_{2}, a_{3}+b_{3}\right)$

2) $A-B=\left(a_{1}-b_{3}, a_{2}-b_{2}, a_{3}-b_{1}\right)$

3) $A \times k=\left(k \times a_{1}, k \times a_{2}, k \times a_{3}\right)$; for $k>0$.

4) $A \times B=\left(a_{1} \times b_{1}, a_{2} \times b_{2}, a_{3} \times b_{3}\right)$; for $R^{+}$.

5) $A \div B=\left(a_{1} \div b_{3}, a_{2} \div b_{2}, a_{3} \div b_{1}\right)$; for $R^{+}$.

Note that other operations could be studied $[13,17,20]$ but in this paper we focus on these ones.

\subsection{The uncertain generalized weighted average}

The uncertain generalized weighted average (UGWA) is an aggregation operator that generalizes the uncertain weighted average (UWA) operator by using generalized means. It is defined as follows.

Definition 1. Let $\Omega$ be the set of interval numbers. An UGWA operator of dimension $n$ is a mapping $U G W A$ : $\Omega^{n} \rightarrow \Omega$ that has an associated weighting vector $W$ of dimension $n$ with $\sum_{i=1}^{n} \tilde{w}_{i}=1$ and $\tilde{w}_{i} \in[0,1]$ such that:

$$
\operatorname{UGWA}\left(\tilde{a}_{1}, \tilde{a}_{2}, \ldots, \tilde{a}_{n}\right)=\left(\sum_{i=1}^{n} \tilde{w}_{i} \tilde{a}_{i}^{\lambda}\right)^{1 / \lambda}
$$

where each $\tilde{a}_{i}$ is an interval number, and $\lambda$ is a parameter such that $\lambda \in(-\infty, \infty)-\{0\}$.

It includes a wide range of particular cases, such as the UWA (when $\lambda=1$ ), the uncertain weighted geometric average (UWGA) (when $\lambda \rightarrow 0$ ), the uncertain weighted quadratic average (UWQA) (when $\lambda=2$ ) and the uncertain weighted harmonic average (UWHA) (when $\lambda=-1$ ).

\subsection{The probabilistic weighted average}

The probabilistic weighted averaging (PWA) operator [7] is an aggregation operator that unifies the probability and the weighted average in the same formulation considering the degree of importance that each concept has in the aggregation. It is defined as follows.

Definition 2. A PWA operator of dimension $n$ is a mapping $P W A: R^{n} \rightarrow R$ such that:

$$
P W A\left(a_{1}, \ldots, a_{n}\right)=\sum_{j=1}^{n} \hat{v}_{i} a_{i}
$$

where the $a_{i}$ are the argument variables, each argument $a_{i}$ has an associated weight (WA) $v_{i}$ with $\sum_{i=1}^{n} v_{i}=1$ and $v_{i} \in[0,1]$, and a probabilistic weight $p_{i}$ with $\sum_{i=1}^{n} p_{i}=1$ and $p_{i} \in[0,1], \hat{v}_{i}=\beta p_{i}+(1-\beta) v_{i}$ with $\beta$ $\in[0,1]$ and $\hat{v}_{i}$ is the weight that unifies probabilities and WAs in the same formulation.

Note that if $\beta=1$, we get the probabilistic aggregation and if $\beta=0$, the weighted average.

\section{The uncertain generalized probabilistic weighted average}

The uncertain generalized probabilistic weighted averaging (UGPWA) operator is an aggregation operator that unifies the probability and the weighted average in the same formulation considering the degree of importance that each concept has in the aggregation. Its main advantage is that it can assess uncertain environments that can not be assessed with exact numbers but it is possible to use interval numbers. Thus, we can analyze the imprecise information considering the minimum and the maximum result that can occur in each situation. Moreover, The UGPWA operator uses generalized means in order to provide a more general formulation that includes a wide range of particular cases such as the uncertain PWA (UPWA), the uncertain probabilistic weighted geometric average (UPGWA), the uncertain probabilistic weighted harmonic average (UPHWA), the uncertain probabilistic weighted quadratic average (UPQWA), and a lot of other cases. Note that in this aggregation, we unify the UGWA with the uncertain generalized probabilistic aggregation (UGPA) and we are able to include other unifications including the uncertain weighted geometric average (UWGA) with the uncertain probabilistic geometric average (UPGA) and the uncertain weighted quadratic average (UWQA) with the uncertain probabilistic quadratic average (UPQA). It is defined as follows.

Definition 3. Let $\Omega$ be the set of interval numbers. An UGPWA operator is a mapping UGPWA: $\Omega^{n} \rightarrow \Omega$ of dimension $n$, if it has an associated probabilistic vector $P$, with $\sum_{i=1}^{n} \tilde{p}_{i}=1$ and $\tilde{p}_{i} \in[0,1]$ and a weighting vector $V$ that affects the weighted average, with $\sum_{i=1}^{n} \tilde{v}_{i}=1$ and $\tilde{v}_{i} \in[0,1]$, such that: 


$$
\begin{aligned}
\operatorname{UGPWA}\left(\tilde{a}_{1}, \tilde{a}_{2}, \ldots, \tilde{a}_{n}\right)= \\
=\tilde{\beta}\left(\sum_{j=1}^{n} \tilde{p}_{i} \tilde{a}_{i}^{\lambda}\right)^{1 / \lambda}+(1-\tilde{\beta})\left(\sum_{i=1}^{n} \tilde{v}_{i} \tilde{a}_{i}^{\sigma}\right)^{1 / \sigma}
\end{aligned}
$$

where the $\tilde{a}_{i}$ are the argument variables represented in the form of interval numbers, $\tilde{\beta} \in[0,1]$ and $\lambda$ and $\sigma$ are a parameter such that $\lambda, \sigma \in(-\infty, \infty)-\{0\}$.

Note that if the weighting vector of probabilities or WAs is not normalized, i.e., $P=\sum_{i=1}^{n} p_{i} \neq 1$, or $V=\sum_{i=1}^{n} v_{i} \neq 1$, then, the UGPWA operator is expressed as follows:

$$
\begin{aligned}
U G P W A & \left(\tilde{a}_{1}, \tilde{a}_{2}, \ldots, \tilde{a}_{n}\right)= \\
& =\frac{\tilde{\beta}}{\tilde{P}}\left(\sum_{j=1}^{n} \tilde{p}_{i} \tilde{a}_{i}^{\lambda}\right)^{1 / \lambda}+\frac{(1-\tilde{\beta})}{\tilde{V}}\left(\sum_{i=1}^{n} \tilde{v}_{i} \tilde{a}_{i}^{\sigma}\right)^{1 / \sigma}
\end{aligned}
$$

Note that it is possible to use a wide range of interval numbers in the aggregation process such as the 2-tuples, the triplets and the quadruplets. Moreover, note that sometimes it is not clear how to reorder the interval numbers. Therefore, it is necessary to establish a criterion for ranking interval numbers. For simplicity, we recommend the following method.

- For 2-tuples, calculate the arithmetic mean of the interval, with $\left(a_{1}+a_{2}\right) / 2$.

- For 3-tuples and above, calculate a weighted average that yields more importance to the central values. That is, for 3-tuples, $\left(a_{1}+3 a_{2}+a_{3}\right) / 5$.

- For 4-tuples, we calculate: $\left(a_{1}+3 a_{2}+3 a_{3}+a_{4}\right) / 8$.

- And so on.

In the case of a tie between the intervals, we select the interval with the lowest difference, i.e., $\left(a_{2}-a_{1}\right)$. For 3-tuples and above odd-tuples, we select the interval with the highest central value. Note that for 4-tuples and above even-tuples, we must calculate the average of the central values following the initial criteria.

The UGPWA is bounded, idempotent and monotonic. It is bounded because the UGPWA aggregation is delimitated by the minimum and the maximum. That is, $\operatorname{Min}\left\{\tilde{a}_{i}\right\} \leq U G P W A\left(\tilde{a}_{1}, \tilde{a}_{2}, \ldots, \tilde{a}_{n}\right) \leq \operatorname{Max}\left\{\tilde{a}_{i}\right\}$. It is idempotent because if $\tilde{a}_{i}=a$, for all $i$, then, UGPWA $\left(\tilde{a}_{1}, \tilde{a}_{2}, \ldots, \tilde{a}_{n}\right)=a$. It is monotonic because if $\tilde{a}_{i} \geq u_{i}$, for all $i$, then, $U G P W A\left(\tilde{a}_{1}, \tilde{a}_{2}, \ldots, \tilde{a}_{n}\right) \geq \operatorname{UGPWA}\left(u_{1}, u_{2}\right.$, $\left.\ldots, u_{n}\right)$.

A further interesting issue to analyze are the measures for characterizing the weighting vector $\hat{V}$. For example, the entropy of dispersion [15,18,22] measures the amount of information being used in the aggregation. For the UGPWA operator, it is defined as follows.

$$
H(\hat{V})=-\left(\tilde{\beta} \sum_{i=1}^{n} \tilde{v}_{i} \ln \left(\tilde{v}_{i}\right)+(1-\tilde{\beta}) \sum_{i=1}^{n} \tilde{p}_{i} \ln \left(\tilde{p}_{i}\right)\right)
$$

Note that $\tilde{p}_{i}$ is the $i$ th weight of the UGPA aggregation and $\tilde{v}_{i}$ the $i$ th weight of the UGWA aggregation. As we can see, if $\widetilde{\beta}=1$ or $\tilde{\beta}=0$, we get the classical Shannon entropy of dispersion [18] by using interval numbers.

The UGPWA operator can be further generalized by using quasi-arithmetic means [2,13] obtaining the uncertain quasi-arithmetic PWA (Quasi-UPWA) operator. It is defined as follows.

Definition 4. Let $\Omega$ be the set of interval numbers. A Quasi-UPWA operator is a mapping QUPWA: $\Omega^{n} \rightarrow \Omega$ of dimension $n$, if it has an associated probabilistic vector $P$, with $\sum_{i=1}^{n} \tilde{p}_{i}=1$ and $\tilde{p}_{i} \in[0,1]$ and a weighting vector $V$ that affects the weighted average, with $\sum_{i=1}^{n} \tilde{v}_{i}=1$ and $\tilde{v}_{i} \in[0,1]$, such that:

$$
\begin{aligned}
& U G P W A\left(\tilde{a}_{1}, \tilde{a}_{2}, \ldots, \tilde{a}_{n}\right)= \\
& =\tilde{\beta}^{-1}\left(\sum_{j=1}^{n} \tilde{p}_{i} g\left(\tilde{a}_{i}\right)\right)+(1-\tilde{\beta}) h^{-1}\left(\sum_{i=1}^{n} \tilde{v}_{i} h\left(\tilde{a}_{i}\right)\right)
\end{aligned}
$$

where the $\tilde{a}_{i}$ are the argument variables represented in the form of interval numbers, $\widetilde{\beta} \in[0,1]$ and $g$ and $h$ are strictly continuous monotonic functions.

As we can see, if $g(a)=a^{\lambda}$ and $h(a)=a^{\sigma}$, the QuasiUPWA operator becomes the UGPWA operator.

\section{Families of UGPWA operators}

The UGPWA operator includes a wide range of particular cases that are useful in some specific situations.

Remark 1. If $\beta=0$, we obtain the uncertain generalized weighted average (UGWA) and if $\widetilde{\beta}=1$, the uncertain generalized probabilistic aggregation (UGPA). Note that it is possible to consider partial cases such as $\tilde{\beta}=$ $(0.8,1)$ where for some part of the interval we get the UGPA (or the UGWA) but not in the whole interval.

Remark 2. If $\tilde{p}_{i}=1 / n$ and $\tilde{v}_{i}=1 / n$, for all $i$, then, we get the uncertain generalized average (UGA). Note that the UGM is also found if $\tilde{\beta}=1$ and $\tilde{p}_{i}=1 / n$, for all $i$, and if $\tilde{\beta}=0$ and $\tilde{v}_{i}=1 / n$, for all $i$.

Remark 3. If $\tilde{v}_{i}=1 / n$, for all $i$, we get the uncertain generalized arithmetic probabilistic aggregation (UGAPA).

$$
\begin{aligned}
U G A-P A & \left(\tilde{a}_{1}, \ldots, \tilde{a}_{n}\right)= \\
= & \tilde{\beta}\left(\sum_{i=1}^{n} \tilde{p}_{i} \tilde{a}_{i}^{\lambda}\right)^{1 / \lambda}+(1-\tilde{\beta})\left(\frac{1}{n} \sum_{i=1}^{n} \tilde{a}_{i}^{\sigma}\right)^{1 / \sigma}
\end{aligned}
$$

Remark 4. If $\tilde{p}_{i}=1 / n$, for all $i$, we get the uncertain generalized arithmetic WA (UGA-WA). 


$$
\begin{aligned}
& U G A-W A\left(\tilde{a}_{1}, \ldots, \tilde{a}_{n}\right)= \\
&=\tilde{\beta}\left(\frac{1}{n} \sum_{i=1}^{n} \tilde{a}_{i}^{\lambda}\right)^{1 / \lambda}+(1-\tilde{\beta})\left(\sum_{i=1}^{n} \tilde{v}_{i} \tilde{a}_{i}^{\sigma}\right)^{1 / \sigma}
\end{aligned}
$$

Theorem 1. If the interval numbers are reduced to the usual exact numbers, then, the UGPWA operator becomes the generalized PWA (GPWA) operator.

Proof. Assume a quadruplet $=\left(a_{1}, a_{2}, a_{3}, a_{4}\right)$. If $a_{1}=a_{2}$ $=a_{3}=a_{4}$, then $\left(a_{1}, a_{2}, a_{3}, a_{4}\right)=a$, thus, we get the GPWA operator.

Remark 5. Note that if the available information is given in different types of interval numbers, then, we have to adapt them to the same structure. Thus, we have to construct an interval that includes all the other ones. For example, if we have one interval with 2-tuples and another one with triplets, then, we have to convert both of them to quadruplets. The 2-tuple is constructed as follows: $\left[a_{1}, a_{2}\right]=\left[a_{1}, a_{1}, a_{2}, a_{2}\right]$ and the triplet in the following way: $\left[a_{1}, a_{2}, a_{3}\right]=\left[a_{1}, a_{2}, a_{2}, a_{3}\right]$.

If we analyze different values of the parameter $\lambda$, we obtain another group of particular cases such as the usual UPWA operator, the UPGWA operator, the UPQWA operator and the UPHWA operator.

Remark 6. When $\lambda=\sigma=1$, the UGPWA operator becomes the UPWA operator.

$$
\operatorname{UGPWA}\left(\tilde{a}_{1}, \ldots, \tilde{a}_{n}\right)=\tilde{\beta} \sum_{i=1}^{n} \tilde{p}_{i} \tilde{a}_{i}+(1-\tilde{\beta}) \sum_{i=1}^{n} \tilde{v}_{i} \tilde{a}_{i}
$$

Note that if $p_{i}=1 / n$, for all $\tilde{a}_{i}$, we get the uncertain arithmetic weighted average (UAWA) and if $v_{i}=1 / n$, for all $\tilde{a}_{i}$, the uncertain arithmetic probabilistic aggregation (UAPA) operator.

Remark 7. When $\lambda \rightarrow 0$ and $\sigma \rightarrow 0$, the UGPWA operator becomes the uncertain probabilistic weighted geometric averaging (UPWGA) operator.

$$
\operatorname{UGPWA}\left(\tilde{a}_{1}, \ldots, \tilde{a}_{n}\right)=\tilde{\beta} \prod_{i=1}^{n} \tilde{a}_{i}^{\tilde{p}_{i}}+(1-\tilde{\beta}) \prod_{i=1}^{n} \tilde{a}_{i} \tilde{v}_{i}
$$

If $p_{i}=1 / n$, for all $\tilde{a}_{i}$, we obtain the uncertain geometric averaging weighted geometric average (UGAWGA) and if $v_{i}=1 / n$, for all $\tilde{a}_{i}$, we get the uncertain geometric averaging probabilistic geometric average (UGAPGA) operator. Note that if $\beta=1$, we get the uncertain probabilistic geometric aggregation (UPGA).

Remark 8. When $\lambda=\sigma=-1$, we get the uncertain probabilistic weighted harmonic averaging (UPWHA) operator.

$$
U G P W A\left(\tilde{a}_{1}, \ldots, \tilde{a}_{n}\right)=\tilde{\beta} \frac{1}{\sum_{i=1}^{n} \frac{\tilde{p}_{i}}{\tilde{a}_{i}}}+(1-\tilde{\beta}) \frac{1}{\sum_{i=1}^{n} \frac{\hat{v}_{i}}{\tilde{a}_{i}}}
$$

If $p_{i}=1 / n$, for all $\tilde{a}_{i}$, we form the uncertain harmonic averaging weighted harmonic average (UHAWHA) and if $v_{i}=1 / n$, for all $\tilde{a}_{i}$, the uncertain harmonic averaging probabilistic harmonic average (UHAPHA) operator. Note that if $\beta=1$, we get the uncertain probabilistic harmonic aggregation (UPHA).

Remark 9. When $\lambda=\sigma=2$, we get the uncertain probabilistic weighted quadratic averaging (UPWQA) operator.

$$
\begin{aligned}
& U G P W A\left(\tilde{a}_{1}, \ldots, \tilde{a}_{n}\right)= \\
&=\tilde{\beta}\left(\sum_{i=1}^{n} \tilde{p}_{i} \tilde{a}_{i}^{2}\right)^{1 / 2}+(1-\tilde{\beta})\left(\sum_{i=1}^{n} \hat{v}_{i} \tilde{a}_{i}^{2}\right)^{1 / 2}
\end{aligned}
$$

If $p_{i}=1 / n$, for all $\tilde{a}_{i}$, we get the uncertain probabilistic quadratic averaging weighted quadratic average (UPQAWQA) and if $v_{i}=1 / n$, for all $\tilde{a}_{i}$, we get the uncertain quadratic averaging probabilistic quadratic average (UQAPQA) operator. Note that if $\beta=1$, we get the uncertain probabilistic quadratic aggregation (UPQA).

Moreover, we can also consider situations where $\lambda \neq$ $\sigma$. For example, we can form the following aggregation operators:

If $\lambda=1$ and $\sigma=2$, we get the uncertain probabilistic quadratic weighted averaging (UPQWA) operator.

$$
f\left(\tilde{a}_{1}, \ldots, \tilde{a}_{n}\right)=\tilde{\beta} \sum_{i=1}^{n} \tilde{p}_{i} \tilde{a}_{i}+(1-\tilde{\beta})\left(\sum_{i=1}^{n} \tilde{v}_{i} \tilde{a}_{i}^{2}\right)^{1 / 2}
$$

If $\lambda=1$ and $\sigma=3$, we form the uncertain probabilistic cubic weighted averaging (UPCWA) operator.

$$
f\left(\tilde{a}_{1}, \ldots, \tilde{a}_{n}\right)=\tilde{\beta} \sum_{i=1}^{n} \tilde{p}_{i} \tilde{a}_{i}+(1-\tilde{\beta})\left(\sum_{i=1}^{n} \tilde{v}_{i} \tilde{a}_{i}^{3}\right)^{1 / 3}
$$

If $\lambda=2$ and $\sigma=1$, we get the uncertain quadratic probabilistic weighted averaging (UQPWA) operator.

$$
f\left(\tilde{a}_{1}, \ldots, \tilde{a}_{n}\right)=\tilde{\beta}\left(\sum_{i=1}^{n} \tilde{p}_{i} \tilde{a}_{i}^{2}\right)^{1 / 2}+(1-\tilde{\beta}) \sum_{i=1}^{n} \tilde{v}_{i} \tilde{a}_{i}
$$

If $\lambda=2$ and $\sigma=3$, we obtain the uncertain quadratic probabilistic cubic weighted averaging (UQPCWA) operator.

$$
\begin{aligned}
f\left(\tilde{a}_{1}, \ldots, \tilde{a}_{n}\right) & = \\
& =\tilde{\beta}\left(\sum_{i=1}^{n} \tilde{p}_{i} \tilde{a}_{i}^{2}\right)^{1 / 2}+(1-\tilde{\beta})\left(\sum_{i=1}^{n} \hat{v}_{i} \tilde{a}_{i}^{3}\right)^{1 / 3}
\end{aligned}
$$


Further situations could be considered by using mixture operators and norm aggregations [25].

\section{Application in decision making with the theory of expertons}

In the following, we develop an uncertain multiperson decision making problem by using the theory of expertons [5-6]. Note that an experton is an extension of the concept of probabilistic set [4] for uncertain environments that can not be assessed with exact numbers but it is possible to use interval numbers. It is very useful in group decision making problems because we can deal with the opinion of several experts in the analysis in a more efficient way since we can assess the information showing various details on their information and the general tendency of the opinion of the group. Note that in the literature, we can find a lot of other decision making approaches $[3,14,16]$.

Assume a country that it is analyzing its general strategy for the next year is planning its monetary policy and consider three alternatives.

- $A_{1}$ : Develop an expansive monetary policy.

- $A_{2}$ : Do not make any change.

- $A_{3}$ : Develop a contractive monetary policy.
In order to evaluate these strategies, the country is assessed with the opinion of five groups of experts. They consider that the key factor for the determination of the expected benefits with each monetary policy is the economic situation for the next year. They have summarized the possible scenarios in the following ones:

- $S_{1}$ : Bad economic situation.

- $S_{2}$ : Regular economic situation.

- $S_{3}$ : Good economic situation.

- $S_{4}$ : Very good economic situation.

Each group of experts evaluates the expected benefits of the country according to the economic situation for the next year. They give their opinion in the interval $[0$, 1] being 0 the lowest expected benefits (or highest loses) and 1 the highest ones. As the available information is very uncertain, the experts provide their information with interval numbers represented in the form of triplets. The results are shown in Tables 1, 2, 3, 4 and 5.

With this information, we construct the expertons. Note that when using triplets, we refer to them as mexpertons [6]. The results are shown in Table 6.

Table 1: Expert 1

\begin{tabular}{ccccc}
\hline & $S_{1}$ & $S_{2}$ & $S_{3}$ & $S_{4}$ \\
\hline$A_{1}$ & {$[0.2,0.3,0.4]$} & {$[0.6,0.7,0.8]$} & {$[0.2,0.3,0.4]$} & {$[0.5,0.6,0.7]$} \\
$A_{2}$ & 0.5 & {$[0.3,0.4,0.5]$} & {$[0.4,0.5,0.7]$} & {$[0.4,0.5,0.6]$} \\
$A_{3}$ & {$[0.1,0.2,0.4]$} & {$[0.6,0.8,0.9]$} & {$[0.8,0.9,1]$} & {$[0.7,0.8,0.9]$} \\
\hline
\end{tabular}

Table 2: Expert 2.

\begin{tabular}{ccccc}
\hline & $S_{1}$ & $S_{2}$ & $S_{3}$ & $S_{4}$ \\
\hline$A_{1}$ & {$[0.2,0.3,0.4]$} & {$[0.2,0.3,0.5]$} & {$[0.3,0.4,0.6]$} & {$[0.3,0.5,0.7]$} \\
$A_{2}$ & {$[0.7,0.8,1]$} & {$[0.3,0.4,0.6]$} & {$[0.1,0.2,0.3]$} & {$[0.4,0.6,0.7]$} \\
$A_{3}$ & {$[0.6,0.7,0.8]$} & {$[0.5,0.6,0.7]$} & {$[0.4,0.5,0.6]$} & {$[0.2,0.3,0.5]$} \\
\hline
\end{tabular}

Table 3: Expert 3.

\begin{tabular}{ccccc}
\hline & $S_{1}$ & $S_{2}$ & $S_{3}$ & $S_{4}$ \\
\hline$A_{1}$ & {$[0.2,0.3,0.4]$} & {$[0.5,0.6,0.7]$} & {$[0.3,0.5,0.6]$} & {$[0.4,0.6,0.7]$} \\
$A_{2}$ & {$[0.1,0.2,0.4]$} & 0.7 & {$[0.6,0.7,0.8]$} & {$[0.7,0.8,0.9]$} \\
$A_{3}$ & {$[0.6,0.8,0.9]$} & {$[0.2,0.4,0.6]$} & {$[0.4,0.5,0.6]$} & {$[0.5,0.6,0.7]$} \\
\hline
\end{tabular}

Table 4: Expert 4.

\begin{tabular}{lcccc}
\hline & $S_{1}$ & $S_{2}$ & $S_{3}$ & $S_{4}$ \\
\hline$A_{1}$ & {$[0.2,0.3,0.4]$} & {$[0.5,0.6,0.7]$} & {$[0.2,0.3,0.4]$} & {$[0.4,0.6,0.7]$} \\
$A_{2}$ & {$[0.2,0.4,0.5]$} & {$[0.2,0.3,0.4]$} & 0.4 & {$[0.7,0.8,0.9]$} \\
$A_{3}$ & {$[0.3,0.4,0.6]$} & 0.6 & {$[0.3,0.4,0.7]$} & {$[0.2,0.3,0.6]$} \\
\hline
\end{tabular}

Table 5: Expert 5.

\begin{tabular}{ccccc}
\hline & $S_{1}$ & $S_{2}$ & $S_{3}$ & $S_{4}$ \\
\hline$A_{1}$ & {$[0.1,0.4,0.5]$} & {$[0.6,0.7,0.8]$} & {$[0.1,0.3,0.4]$} & {$[0.5,0.6,0.8]$} \\
$A_{2}$ & {$[0.5,0.6,0.8]$} & {$[0.5,0.6,0.8]$} & {$[0.2,0.3,0.4]$} & {$[0.3,0.4,0.5]$} \\
$A_{3}$ & {$[0.3,0.5,0.6]$} & {$[0.1,0.2,0.3]$} & {$[0.4,0.6,0.7]$} & {$[0.5,0.6,0.7]$} \\
\hline
\end{tabular}


Table 6: Expertons for each strategy and state of nature.

\begin{tabular}{|c|c|c|c|c|c|c|c|c|c|c|c|c|c|c|c|c|}
\hline & \multicolumn{4}{|c|}{$S_{1}$} & \multicolumn{4}{|c|}{$S_{2}$} & \multicolumn{4}{|c|}{$S_{3}$} & \multicolumn{4}{|c|}{$S_{4}$} \\
\hline \multirow{11}{*}{$A_{1}$} & 0 & 1 & 1 & 1 & \multirow{11}{*}{$\begin{array}{c}0 \\
0.1 \\
0.2 \\
0.3 \\
0.4 \\
0.5 \\
0.6 \\
0.7 \\
0.8 \\
0.9 \\
1\end{array}$} & 1 & 1 & 1 & \multirow{11}{*}{$\begin{array}{c}0 \\
0.1 \\
0.2 \\
0.3 \\
0.4 \\
0.5 \\
0.6 \\
0.7 \\
0.8 \\
0.9 \\
1\end{array}$} & 1 & 1 & 1 & \multirow{11}{*}{$\begin{array}{c}0 \\
0.1 \\
0.2 \\
0.3 \\
0.4 \\
0.5 \\
0.6 \\
0.7 \\
0.8 \\
0.9 \\
1\end{array}$} & 1 & 1 & 1 \\
\hline & 0.1 & 1 & 1 & 1 & & 1 & 1 & 1 & & & 1 & 1 & & 1 & 1 & 1 \\
\hline & 0.2 & 0.8 & 1 & 1 & & 1 & 1 & 1 & & 0.8 & 1 & 1 & & 1 & 1 & 1 \\
\hline & 0.3 & 0 & 1 & 1 & & 0.8 & 1 & 1 & & 0.4 & 1 & 1 & & 1 & 1 & 1 \\
\hline & 0.4 & 0 & 0.2 & 1 & & 0.8 & 0.8 & 1 & & 0 & 0.4 & 1 & & 0.8 & 1 & 1 \\
\hline & 0.5 & 0 & 0 & 0.2 & & 0.8 & 0.8 & 1 & & 0 & 0.2 & 0.4 & & 0.4 & 1 & 1 \\
\hline & 0.6 & 0 & 0 & 0 & & 0.4 & 0.8 & 0.8 & & 0 & 0 & 0.4 & & 0 & 0.8 & 1 \\
\hline & 0.7 & 0 & 0 & 0 & & 0 & 0.4 & 0.8 & & 0 & 0 & 0 & & 0 & 0 & 1 \\
\hline & 0.8 & 0 & 0 & 0 & & 0 & 0 & 0.4 & & 0 & 0 & 0 & & 0 & 0 & 0.2 \\
\hline & 0.9 & 0 & 0 & 0 & & 0 & 0 & 0 & & 0 & 0 & 0 & & 0 & 0 & 0 \\
\hline & 1 & 0 & 0 & 0 & & 0 & 0 & 0 & & 0 & 0 & 0 & & 0 & 0 & 0 \\
\hline \multirow{11}{*}{$A_{2}$} & 0 & 1 & 1 & 1 & 0 & 1 & 1 & 1 & \multirow{11}{*}{$\begin{array}{c}0 \\
0.1 \\
0.2 \\
0.3 \\
0.4 \\
0.5 \\
0.6 \\
0.7 \\
0.8 \\
0.9 \\
1\end{array}$} & 1 & 1 & 1 & \multirow{11}{*}{$\begin{array}{c}0 \\
0.1 \\
0.2 \\
0.3 \\
0.4 \\
0.5 \\
0.6 \\
0.7 \\
0.8 \\
0.9 \\
1\end{array}$} & 1 & 1 & 1 \\
\hline & 0.1 & 1 & 1 & 1 & 0.1 & 1 & 1 & 1 & & 1 & 1 & 1 & & 1 & 1 & 1 \\
\hline & 0.2 & 0.8 & 1 & 1 & 0.2 & 1 & 1 & 1 & & 0.8 & 1 & 1 & & 1 & 1 & 1 \\
\hline & 0.3 & 0.6 & 0.8 & 1 & 0.3 & 0.8 & 1 & 1 & & 0.6 & 0.8 & 1 & & 1 & 1 & 1 \\
\hline & 0.4 & 0.6 & 0.8 & 1 & 0.4 & 0.4 & 0.8 & 1 & & 0.6 & 0.6 & 0.8 & & 0.8 & 1 & 1 \\
\hline & 0.5 & 0.6 & 0.6 & 0.8 & 0.5 & 0.4 & 0.4 & 0.8 & & 0.2 & 0.4 & 0.4 & & 0.4 & 0.8 & 1 \\
\hline & 0.6 & 0.2 & 0.4 & 0.4 & 0.6 & 0.2 & 0.4 & 0.6 & & 0.2 & 0.2 & 0.4 & & 0.4 & 0.6 & 0.8 \\
\hline & 0.7 & 0.2 & 0.2 & 0.4 & 0.7 & 0.2 & 0.2 & 0.4 & & 0 & 0.2 & 0.4 & & 0.4 & 0.4 & 0.6 \\
\hline & 0.8 & 0 & 0.2 & 0.4 & 0.8 & 0 & 0 & 0.2 & & 0 & 0 & 0.2 & & 0 & 0.4 & 0.4 \\
\hline & 0.9 & 0 & 0 & 0.2 & 0.9 & 0 & 0 & 0 & & 0 & 0 & 0 & & 0 & 0 & 0.4 \\
\hline & 1 & 0 & 0 & 0.2 & 1 & 0 & 0 & 0 & & 0 & 0 & 0 & & 0 & 0 & 0 \\
\hline \multirow{11}{*}{$A_{3}$} & 0 & 1 & 1 & 1 & 0 & 1 & 1 & 1 & \multirow{11}{*}{$\begin{array}{c}0 \\
0.1 \\
0.2 \\
0.3 \\
0.4 \\
0.5 \\
0.6 \\
0.7 \\
0.8 \\
0.9 \\
1\end{array}$} & 1 & 1 & 1 & \multirow{11}{*}{$\begin{array}{c}0 \\
0.1 \\
0.2 \\
0.3 \\
0.4 \\
0.5 \\
0.6 \\
0.7 \\
0.8 \\
0.9 \\
1\end{array}$} & 1 & 1 & 1 \\
\hline & 0.1 & 1 & 1 & 1 & 0.1 & 1 & 1 & 1 & & 1 & 1 & 1 & & 1 & 1 & 1 \\
\hline & 0.2 & 0.8 & 1 & 1 & 0.2 & 0.8 & 1 & 1 & & 1 & 1 & 1 & & 1 & 1 & 1 \\
\hline & 0.3 & 0.8 & 0.8 & 1 & 0.3 & 0.6 & 0.8 & 1 & & 1 & 1 & 1 & & 0.6 & 1 & 1 \\
\hline & 0.4 & 0.4 & 0.8 & 1 & 0.4 & 0.6 & 0.8 & 0.8 & & 0.8 & 1 & 1 & & 0.6 & 0.6 & 1 \\
\hline & 0.5 & 0.4 & 0.6 & 0.8 & 0.5 & 0.6 & 0.6 & 0.8 & & 0.2 & 0.8 & 1 & & 0.6 & 0.6 & 1 \\
\hline & 0.6 & 0.4 & 0.4 & 0.8 & 0.6 & 0.4 & 0.6 & 0.8 & & 0.2 & 0.4 & 1 & & 0.2 & 0.6 & 0.8 \\
\hline & 0.7 & 0 & 0.4 & 0.4 & 0.7 & 0 & 0.2 & 0.4 & & 0.2 & 0.2 & 0.6 & & 0.2 & 0.2 & 0.6 \\
\hline & 0.8 & 0 & 0.2 & 0.4 & 0.8 & 0 & 0.2 & 0.2 & & 0.2 & 0.2 & 0.2 & & 0 & 0.2 & 0.2 \\
\hline & 0.9 & 0 & 0 & 0.2 & 0.9 & 0 & 0 & 0.2 & & 0 & 0.2 & 0.2 & & 0 & 0 & 0.2 \\
\hline & 1 & 0 & 0 & 0 & 1 & 0 & 0 & 0 & & 0 & 0 & 0.2 & & 0 & 0 & 0 \\
\hline
\end{tabular}

Table 7: Experton for $A_{1}$ and $S_{2}$.

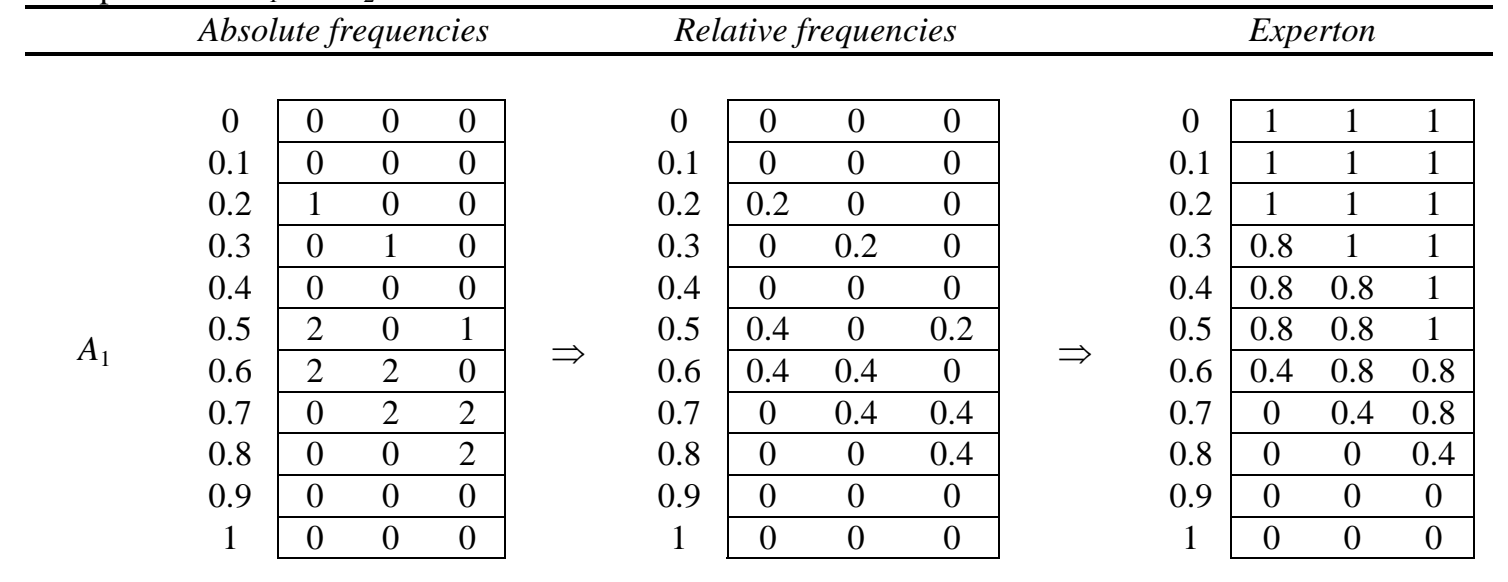


Table 8: Expected value of the expertons for each strategy and state of nature.

\begin{tabular}{ccccc}
\hline & $S_{1}$ & $S_{2}$ & $S_{3}$ & $S_{4}$ \\
\hline$A_{1}$ & {$[0.18,0.32,0.42]$} & {$[0.48,0.58,0.7]$} & {$[0.22,0.36,0.48]$} & {$[0.42,0.58,0.72]$} \\
$A_{2}$ & {$[0.4,0.5,0.6]$} & {$[0.4,0.48,0.6]$} & {$[0.34,0.42,0.52]$} & {$[0.5,0.62,0.72]$} \\
$A_{3}$ & {$[0.38,0.52,0.66]$} & {$[0.4,0.52,0.62]$} & {$[0.46,0.58,0.72]$} & {$[0.42,0.52,0.68]$} \\
\hline
\end{tabular}

Table 9: Aggregated results.

\begin{tabular}{ccccc}
\hline & $U A$ & $U P A$ & $U W A$ & $U P W A$ \\
\hline$A_{1}$ & {$[0.325,0.46,0.58]$} & {$[0.354,0.488,0.612]$} & {$[0.324,0.462,0.584]$} & {$[0.333,0.4698,0.5924]$} \\
$A_{2}$ & {$[0.41,0.505,0.61]$} & {$[0.412,0.506,0.612]$} & {$[0.412,0.508,0.612]$} & {$[0.412,0.5074,0.612]$} \\
$A_{3}$ & {$[0.415,0.535,0.67]$} & {$[0.422,0.538,0.672]$} & {$[0.42,0.538,0.676]$} & {$[0.4206,0.538,0.6748]$} \\
\hline
\end{tabular}

Note that in order to calculate the results shown in Table 6, we use the following methodology presented in Table 7 for the experton $A_{1}$ with $S_{2}$. That is, first we calculate the absolute frequencies (the number of experts that gives each result). Next, we calculate the relative frequencies (we divide the absolute frequencies by the total number of experts) and finally, the accumulated relative frequency of the results (we sum from $\alpha=$ 1 , the relative frequencies in an accumulated way until $\alpha=0$ ) [5-6]. The same should be done for all the other expertons.

Next, we calculate the expected value of the expertons. For doing so, we sum all the levels of membership $\alpha$ excepting the 0 and divide the result by 10 . The results are shown in Table 8.

The results obtained in Table 8 can be used in an aggregation process in order to obtain a single result that permits us to see the expected benefits by using each alternative. Note that in this aggregation process we use several particular cases of the UGPWA operator. In this paper, we consider the UA, the UWA, the UPA and the UPWA operator. We assume that the UWA uses the following weighting vector $V=(0.2,0.2,0.3,0.3)$ with a $70 \%$ of importance and the UPA: $P=(0.1,0.3,0.3$, $0.3)$ with $30 \%$ of importance. The results are shown in Table 9.

As we can see, each particular type of UGPWA operator may lead to different results and decisions. In this example, it seems that $A_{3}$ is the optimal choice.

\section{Conclusions}

We have presented the UGPWA operator. It is an aggregation operator that unifies the uncertain probabilistic aggregation and the UWA operator in the same formulation and considering the degree of importance that each concept has in the aggregation. Thus, it is very useful for uncertain environments where the available information is imprecise and can not be assessed with exact numbers but it is possible to use interval numbers. We have seen that the use of interval numbers permits to represent the information in a more complete way because we can assess the imprecise information by using minimums and maximums that guarantees that the information is contained inside these bounds.

Moreover, by using generalized aggregation operators, we have seen that the UGPWA operator includes a wide range of particular cases including the UPA, the UWA, the UGPA, the UGWA, the PWA, the GPWA, the UGA-PA and the UGA-WA operator. We have further generalized the UGPWA by using quasi-arithmetic means obtaining the Quasi-UPWA operator.

We have studied the applicability of the UGPWA operator in a multi-person decision making problem regarding the selection of monetary policies. We have used the theory of expertons in order to assess the multi-person analysis. Thus, we have represented the information in a more general way that considers the individual opinions inside the group and produces a final representative result. The main advantage of the UGPWA operator is that it can consider a wide range of scenarios by using different particular cases and select the one in closest accordance with the specific interests of the decision maker.

In future research we expect to develop further developments to this approach by adding more characteristics in the analysis such as the use of the ordered weighted averaging (OWA) operator and the unified aggregation operators (UAO). Moreover, we will also use other types of uncertain information such as fuzzy numbers or linguistic variables and other potential applications giving special attention to decision theory and statistics.

\section{References}

[1] G. Beliakov, A. Pradera and T. Calvo, Aggregation Functions: A Guide for Practitioners, Berlin: Springer-Verlag, 2007.

[2] J. Fodor, J.L. Marichal and M. Roubens, Characterization of the ordered weighted averaging operators, IEEE Transactions on Fuzzy Systems, 3: 236240, 1995.

[3] A.M. Gil-Lafuente and J.M. Merigó, Computational Intelligence in Business and Economics, World Scientific, Singapore, 2010.

[4] K. Hirota, Concept of probabilistic sets, Fuzzy Sets and Systems, 5: 31-46, 1981.

[5] A. Kaufmann, Theory of expertons and fuzzy logic, Fuzzy Sets and Systems, 28: 295-304, 1988.

[6] A. Kaufmann and J. Gil-Aluja, Special techniques for the management of experts (In Spanish), Ed. Milladoiro, Vigo, 1993.

[7] J.M. Merigó, The probabilistic weighted averaging operator and its application in decision making, in: Operations Systems Research \& Security of Information, Canada, pp. 55-58, 2009. 
[8] J.M. Merigó, Fuzzy decision making with immediate probabilities, Computers \& Industrial Engineering, 58: 651-657, 2010.

[9] J.M. Merigó and M. Casanovas, Induced aggregation operators in decision making with DempsterShafer belief structure, International Journal of Intelligent Systems, 24: 934-954, 2009.

[10] J.M. Merigó and M. Casanovas, Fuzzy generalized hybrid aggregation operators and its application in decision making, International Journal of Fuzzy Systems, 12: 15-24, 2010.

[11] J.M. Merigó and M. Casanovas, Induced and heavy aggregation operators with distance measures, Journal of Systems Engineering and Electronics, 21: 431-439, 2010.

[12] J.M. Merigó and M. Casanovas, The fuzzy generalized OWA operator and its application in strategic decision making, Cybernetics \& Systems, 41: 359370, 2010.

[13] J.M. Merigó and M. Casanovas, The uncertain induced quasi-arithmetic OWA operator, International Journal of Intelligent Systems, 26: 1-24, 2011.

[14] J.M. Merigó and M. Casanovas, Decision making with distance measures and induced aggregation operators, Computers \& Industrial Engineering, 60: 66-76, 2011.

[15] J.M. Merigó and A.M. Gil-Lafuente, The induced generalized OWA operator, Information Sciences, 179: 729-741, 2009.

[16] J.M. Merigó and A.M. Gil-Lafuente, New decision making techniques and their application in the selection of financial products, Information Sciences, 180: 2085-2094, 2010.

[17] R.E. Moore, Interval Analysis, Prentice Hall, Englewood Cliffs, NJ, 1966.
[18] C.E. Shannon, A mathematical theory of communication, Bell Systems Technical Journal, 27: 379423, 1948.

[19] V. Torra, The weighted OWA operator, International Journal of Intelligent Systems, 12: 153-166, 1997.

[20] Z.S. Xu and Q.L. Da, The uncertain OWA operator, International Journal of Intelligent Systems, 17: 569-575, 2002.

[21] Z.S. Xu and Q.L. Da, An overview of operators for aggregating information, International Journal of Intelligent Systems, 18: 953-968, 2003.

[22] R.R. Yager, On ordered weighted averaging aggregation operators in multi-criteria decision making, IEEE Transactions on Systems, Man Cybernetics B, 18: 183-190, 1988.

[23] R.R. Yager, Families of OWA operators, Fuzzy Sets and Systems, 59: 125-148, 1993.

[24] R.R. Yager, Generalized OWA aggregation operators, Fuzzy Optimization and Decision Making, 3: 93-107, 2004.

[25] R.R. Yager, Norms induced from OWA operators, IEEE Transactions on Fuzzy Systems, 18: 57-66, 2010.

[26] R.R. Yager, K.J. Engemann and D.P. Filev, On the concept of immediate probabilities, International Journal of Intelligent Systems, 10: 373-397, 1995.

[27] R.R. Yager and J. Kacprzyk, The Ordered Weighted Averaging Operators: Theory and Applications. Norwell: Kluwer Academic Publishers, 1997.

[28] L.G. Zhou and H.Y. Chen, Generalized ordered weighted logarithm aggregation operators and their applications to group decision making. International Journal of Intelligent Systems, 25: 683-707, 2010. 